\title{
OsACOS12, an orthologue of Arabidopsis acyl-CoA synthetase5, plays an important role in pollen exine formation and anther development in rice
}

\author{
Yueling Li, Dandan Li, Zongli Guo, Qiangsheng Shi, Shuangxi Xiong, Cheng Zhang, Jun Zhu* and Zhongnan Yang ${ }^{*}$
}

\begin{abstract}
Background: Sporopollenin is a major component of the pollen exine pattern. In Arabidopsis, acyl-CoA synthetase5 (ACOS5) is involved in sporopollenin precursor biosynthesis. In this study, we identified its orthologue, OsACOS12, in rice (Oryza sativa) and compared the functional conservation of ACOS in rice to Arabidopsis.

Results: Sequence analysis showed that OsACOS12 shares $63.9 \%$ amino acid sequence identity with ACOS5. The osacos 12 mutation caused by a pre-mature stop codon in LOC_Os04g24530 exhibits defective sexine resulting in a male sterile phenotype in rice. In situ hybridization shows that OSACOS12 is expressed in tapetal cells and microspores at the transcript level. The localization of OsACOS12-GFP demonstrated that OsACOS12 protein is accumulated in tapetal cells and anther locules. OsACOS12 driven by the ACOS5 promoter could partially restore the male fertility of the acos 5 mutant in Arabidopsis.

Conclusions: OSACOS12 is an orthologue of ACOS5 that is essential for sporopollenin synthesis in rice. ACOS5 and OsACOS12 are conserved for pollen wall formation in monocot and dicot species.
\end{abstract}

Keywords: Oryza sativa, OsACOS12, Male sterility, Pollen exine, Anther cuticle

\section{Background}

Male reproductive development is an essential biological process for the propagation of flowering plants. Pollen development is the major event of male reproduction. Developmental defects leading to male sterility are widely used for hybrid production in agriculture [1]. During pollen development, pollen wall formation is a key process required for pollen viability and male fertility. The pollen wall structure divides into the outer exine and the inner intine. The exine is further divided into a species-specific sexine and a flat nexine [2]. The major composition of the sexine is sporopollenin [3], while the nexine is mainly composed of glycoproteins [4]. The biological function of the sexine layer is to provide an external barrier for adapting the terrestrial environment to ensure microgamete survival in land plants to resist various environmental stresses and microbial

\footnotetext{
* Correspondence: zhujun78@shnu.edu.cn; znyang@shnu.edu.cn College of Life and Environment Sciences, Shanghai Normal University, 100 Guilin Road, Shanghai 200234, China
}

attacks $[5,6]$. The sexine patterning also acts as an important feature of plant taxonomic classifications [7].

Sporopollenin, the major constituent of the sexine, was considered to be a complex polymer primarily composed of long-chain fatty acids, oxygenated aromatic rings and phenylpropanoic acids [8]. The tapetal layer is an essential tissue required for normal sexine development and pollen maturity [9]. Based on cytological and molecular evidence, the material of sporopollenin precursors originate from tapetal cells $[10,11]$. The sporopollenin precursors are inilly deposited at the mould of the sexine to form probaae and protectum structures. After a microspore is eased, the exine structure increases in size with condeposits and polymerization of sporopollenin the decorated sexine pattern is formed [12]. Howto be involved in the complex biochemical pathways of sporopollenin precursor formation, including CYP703A2, 
CYP704B1 and MS2 for fatty-acid-derived compound metabolism, PKSA/B and TKPR1/2 for phenylpropanoids synthesis, and ABCG26 for transportation [13-19]. All these genes are expressed in the tapetal layer at the transcript level. On the protein level, MS2 is localized in tapetal cells, while CYP703A2 is in both tapetal cells and the anther locule $[19,20]$. It is likely that the last several steps of sporopollenin precursor synthesis occur in the locule.

The sporopollenin is a general constituent that has been widely found in moss, ferns, gymnosperms and angiosperms. Sporopollenin synthesis seems to share common metabolic pathways in various species. The tapetum directly provides materials for pollen wall formation. The genetic pathway for tapetum development is generally conserved between rice and Arabidopsis [21]. In rice, several sporopollenin enzymes have been identified [22-25]. The biological functions of these enzymes and the metabolic pathways for sporopollenin synthesis were very conserved between rice and Arabidopsis. However, the anther cuticle had defects in mutations of the rice genes discussed above, whereas homologous mutants in Arabidopsis did not show obvious morphological changes in the anther walls. It was suggested that the lipidic pathway was diversified in rice $[22,25]$.

ACOS5 encodes a fatty acyl-CoA synthetase (ACOS) for sporopollenin precursor synthesis in Arabidopsis [26, 27]. There are nine fatty acyl-CoA synthetase encoding genes in rice [28]. In this study, we characterized an orthologue of Arabidopsis ACOS5 in rice, OsACOS12 (LOC_Os04g24530). A knockout of this gene led to a male sterile phenotype in rice with a defective sexine layer and anther cuticle. The tapetal and anther locule localization of the OsACOS12 protein suggested that there was synchronous biosynthesis and transportation of sporopollenin precursors. The expression of the OsACOS12 gene in the Arabidopsis acos 5 mutant partially restored the male sterile phenotype, which indicated that the acyl-CoA synthetase gene is a conserved function between Arabidopsis and rice.

\section{Results}

OsACOS12 in 0. sativa is an orthologue of ACOS5 in A. thaliana

BLASTP analysis using Arabidopsis ACOS5 protein sequence yield the LOC_Os04g24530 in rice encoding a fatty acyl-CoA synthetase (http://rice.plantbiology.msu.edu/). The sequence of LOC_Os04g24530 also showed the highest sequence similarity with ACOS5 in Arabidopsis genome (63.9 \% amino acid sequence identity, Fig. 1a). LOC_Os04g24530 was designated OsACOS12 previously [26]. The large superfamily of acyl-activating enzymes contains putative motifs for AMP-binding and fatty acidbinding $[29,30]$. These motifs are highly conserved between OsACOS12 and ACOS5 (Fig. 1a). The homologues of OsACOS12 protein have been identified in various plant species by a BLASTP search according to the GenBank database. No orthologue could be identified in the genome of the green alga. Phylogenetic analysis shows OsACOS12 and its homologues formed four distinct clades. The homologues from Physcomitrella and Selaginella formed two distinct clades that diverged early in land plant evolution. The homologues from dicotyledoneae and monocotyledon species form two other clades (Fig. 1b). Sequence analysis demonstrated that ACOS enzymes are apparently present in land plants, which supports a possible role for them in the biosynthesis of sporopollenin, which is the demand for protecting gametophytes to adapt to a land environment.

\section{osacos 12 mutant shows complete male sterility}

To characterize the function of OsACOS12, we obtained an allele of LOC_OsO4g24530 using Targeting Induced Local Lesions In Genomes (Tilling) technology from the ethyl methane sulfonate-induced population of rice Zhonghua11 (O. sativa ssp. Japonica) [31]. Sequence analysis revealed a point mutation from $\mathrm{A}$ to $\mathrm{T}$, in the 1000th base downstream of the start codon of the LOC_Os04g24530 genomic sequence in the mutant. This transition caused a premature termination (AAG-TAG) at the first exon of LOC_OsO4g24530 (Fig. 2a). The osa$\cos 12$ mutant exhibited normal vegetative and spikelet development (Fig. 2c, e). However, the mutant anthers had a white colour without pollen grains inside, which led to complete male sterility (Fig. 2c, f-h). Reciprocal crosses with the wild type indicted that female fertility was not affected in the osacos 12 mutant. The fertile and sterile plants of the $F_{2}$ population segregated with a 3:1 ratio (86:23) indicated that there was a single recessive sporophytic mutation for osacos 12 . To complement the osacos 12 mutant phenotype, the OsACOS12 genomic fragment fused with GFP driven by its own promoter (1428 bp) was constructed and transformed into heterozygous OsACOS12 seeds. Of the 32 transgenic lines, 7 were identified to have a homozygous osacos 12 mutant background (Fig. 2i-j). All of these homozygous lines exhibited normal fertility (Fig. 2d). These results demonstrate that OsACOS12 was responsible for the male fertility of the osacos 12 mutant.

\section{Pollen sexine formation and anther cuticle are defective in osacos 12}

Scanning electron microscopy (SEM) was used to elucidate the abnormal morphological defects of anther development in the osacos12 plant. The osacos12 anther was much shorter and smaller compared to that of the wild type (Fig. 3a-b). In the wild type, the anther surface was covered by cuticle (Fig. 3c), and orbicules were 
$\mathbf{a}$

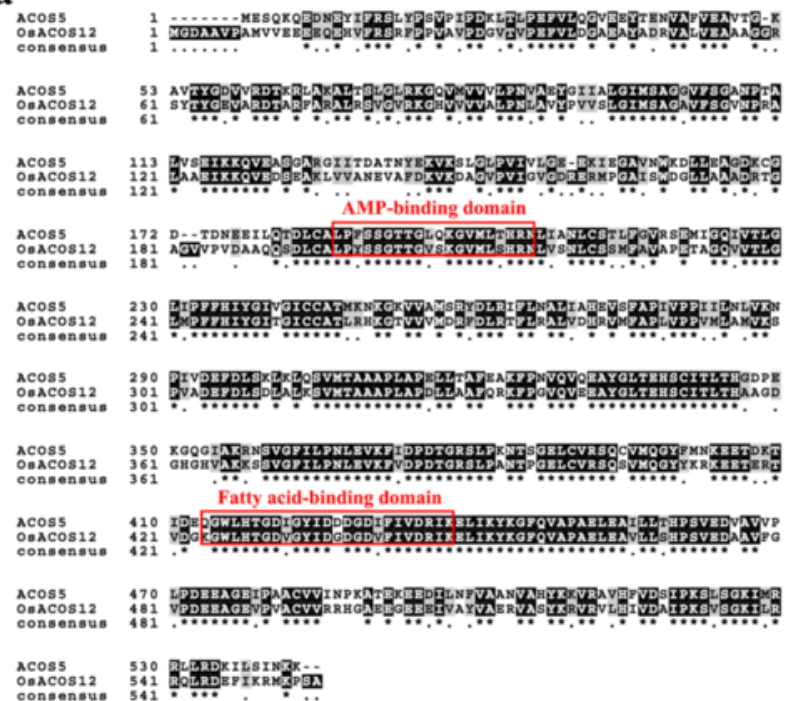

\section{b}

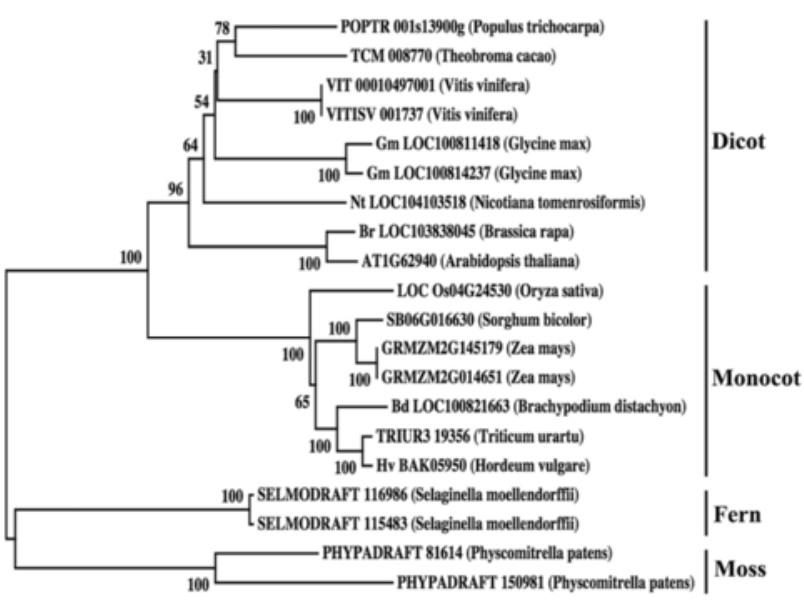

0.05

Fig. 1 OsACOS12 in O. sativa is an orthologue of ACOS5 in Arabidopsis. a Amino acid sequences alignment of OsACOS12 and ACOS5. The sequences were aligned using Clustal W and displayed using BOXSHADE. b A neighbour-joining phylogenetic tree of OsACOS 12 and its orthologues in different species. Bootstrap values are the percentage of 1,000 replicates. The conserved AMP binding domain and fatty acid binding domain are indicated

intensively distributed on the inner surface (Fig. 3d). However, osacos 12 anther surface was lack of these materials and looks smooth (Fig. 3g), and orbicules were barely detected in the inner surface (Fig. 3h). The wild type anther was filled with mature pollen grains (Fig. 3e-f). In contrast, only a few remnants of degenerated pollen could be observed in the osacos 12 anther (Fig. 3i-j). In the complementary transgenic lines, the anther surface and pollen exine were restored (Additional file 1: Figure S1).

We subsequently obtained semi-thin transverse sections to understand the detailed defects of pollen development in osacos12. There were no detectable differences between wild type and osacos 12 during early anther development. The microspore mother cells (MMCs) and tetrads of osa$\cos 12$ appeared to be comparable with the wild type (Fig. 3k-l, p-q). In the wild type, newly released microspores of the wild type were angular in shape (Fig. $3 \mathrm{~m}$ ) and became enlarged and vacuolated (Fig. 3n). In osacos 12 plants, the released microspores contained much less cytoplasm (Fig. 3r) and were degenerated before/during volume enlargement (Fig. 3s). No mature pollen grains were observed in the locule of osacos12 at later stages of anther development (Fig. 3t). The defective phenotype of $o s a \cos 12$ was similar to a cos 5 in Arabidopsis.

To further clarify the details of the abnormal exine development of osacos 12 pollen, anther samples were investigated using transmission electron microscopy (TEM). During the tetrad stage, primexine is formed between callose wall and plasma membrane. It is critical for pollen wall pattern. The primexine formation in osacos12 is consistent with that in wild type at tetrad stage (Fig. $3 \mathrm{u}, \mathrm{x}$ ). At stage 9, the pollen exine in osacos 12 was not as deeply stained as that in the wild type, indicating an abnormal sporopollenin deposition (Fig. 3v, y). At stage 10, the exine layer of microspore was formed with gradually deposition of sporopollenin precursors in the wild type (Fig. 3w). However, in osacos12, no sporopollenin precursor accumulated on the microspore surface resulted in absent exine layer phenotype of collapsed pollen grains (Fig. 3z). Additionally, there were no obvious aberrations in the appearance of the tapetum in the osacos12 mutant (Additional file 2: Figure S2). These observations revealed that the exine formation and cuticle structures of anther epidermis were abnormal in $\operatorname{osacos} 12$.

\section{Defective wax components in osacos 12 anthers}

The defective anther cuticle and sporopollenin in osa$\cos 12$ suggested that the lipidic mechanisms were aberrant in the mutant. To confirm this point, we performed gas chromatography-mass spectrometry (GC-MS) to quantify wax extracts from whole anthers of both the wild type and osacos 12 mutants. The results showed that the total cuticular wax amount was reduced by approximately $42.6 \%$ in the mutant (Fig. 4a), which contributed to the significant reduction of most wax constituents. The components of wax, including long-chain fatty acids (C14 to C26), alkanes (C30 to C36) and alcohols (C28 to C30) were significantly decreased in the osacos 12 mutant (Fig. 4b). Therefore, chemical analysis indicated that 

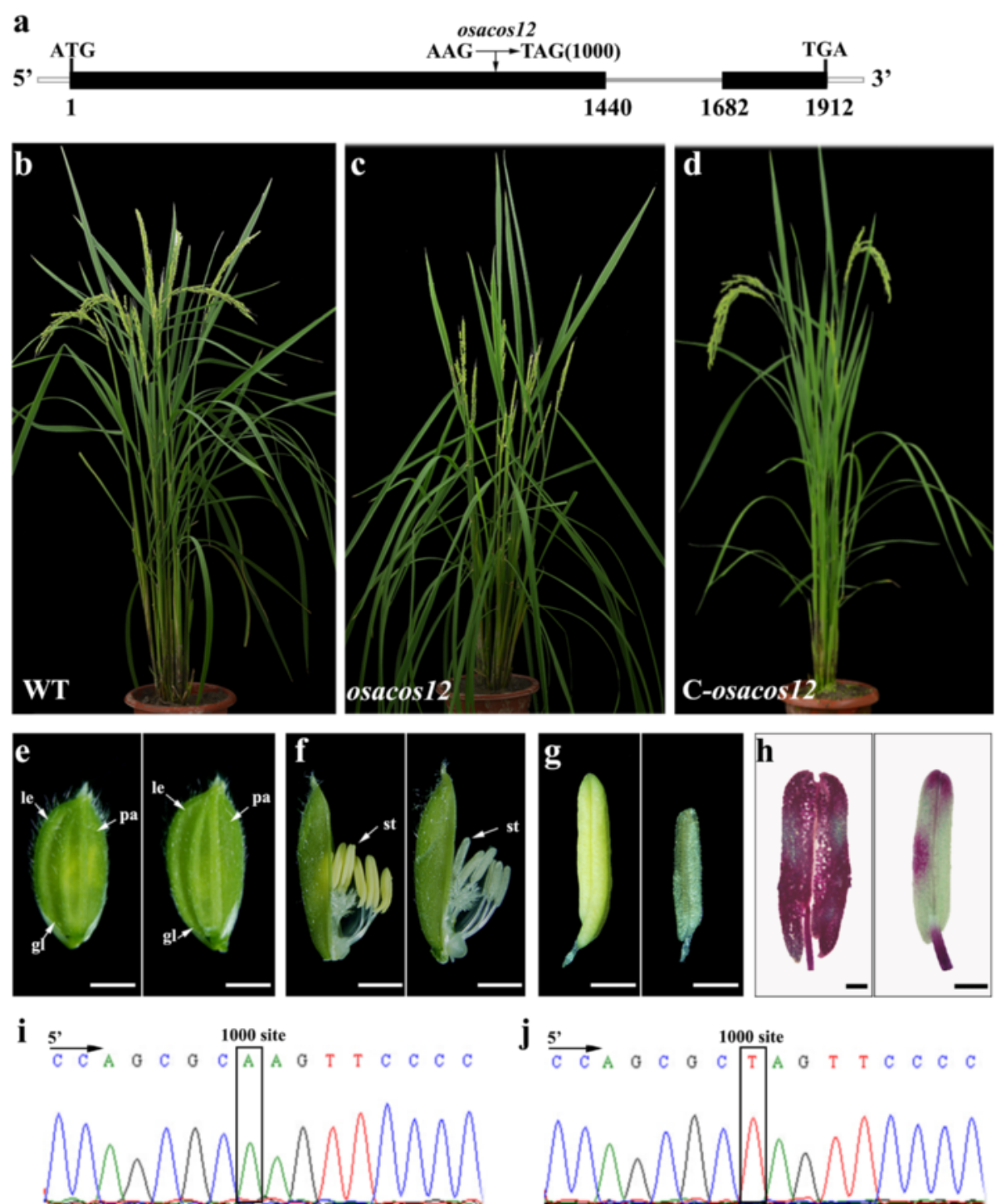

Fig. 2 Isolation of the rice osacos 12 mutant with complete male sterility. a The gene structure and position of the nucleotide change in osacos 12 . The black boxes indicate exons. b-d The wild-type (WT) plant, osacos 12 mutant and complementation plant after the heading stage. e Comparison of the WT plant (left) and osacos 12 mutant panicles (right) at the heading stage. $\mathbf{f}$ The spikelets of the WT plant (left) and an osacos 12 mutant (right) after removing the palea. $\mathbf{g}$ The anther of a WT plant (left) and an osacos 12 mutant (right). $\mathbf{h}$ Alexander staining of the WT plant (left) and an osacos 12 mutant anther (right). $\mathbf{i}$ and $\mathbf{j}$ Identification of the OSACOS12 gene in a WT plant and osacos 12 mutant by sequencing (position 1000). Bars $=100 \mu \mathrm{m}$ in $\mathbf{e - g}$ and $200 \mu \mathrm{m}$ in $\mathbf{h}$

OsACOS12 was involved in the synthesis of lipidic compounds during rice anther development.

\section{OsACOS12 is located in the tapetum and anther locule}

In Arabidopsis, ACOS5 is expressed in tapetal cells and microspores from late stage 5 to stage 8 , as shown through in situ hybridization [26]. To analyse the expression of OsACOS12 in rice, semi-quantitative RTPCR analysis was performed. OsACOS12 expression was detected in the anthers with glume lengths of $2.5 \mathrm{~mm}$ to $4.0 \mathrm{~mm}$ but was not detected in roots, shoots, leaves and palea/lemma (Fig. 5a). This result was further confirmed by quantitative real-time PCR analysis (Fig. 5b). Spatial and temporal expressions of OsACOS12 were detected by an RNA in situ hybridization analysis using an OsACOS12-specific probe. The OsACOS12 was initially expressed in the tapetal layer and microspore mother cells at the beginning of meiosis (Fig. $5 \mathrm{c}-\mathrm{d}$ ). The signal was increased significantly and reached the highest level during the tetrad stage (Fig. 5e-f). At microspores stage, the signal of OsACOS12 transcripts was obviously decreased in the tapetum and microspores (Fig. 5g-h). In the control, only background signal was detected using a sense probe at tetrad stage (Fig. 5i-j).

To understand the expression of OsACOS12 at the protein level, we analysed the GFP signal in the 

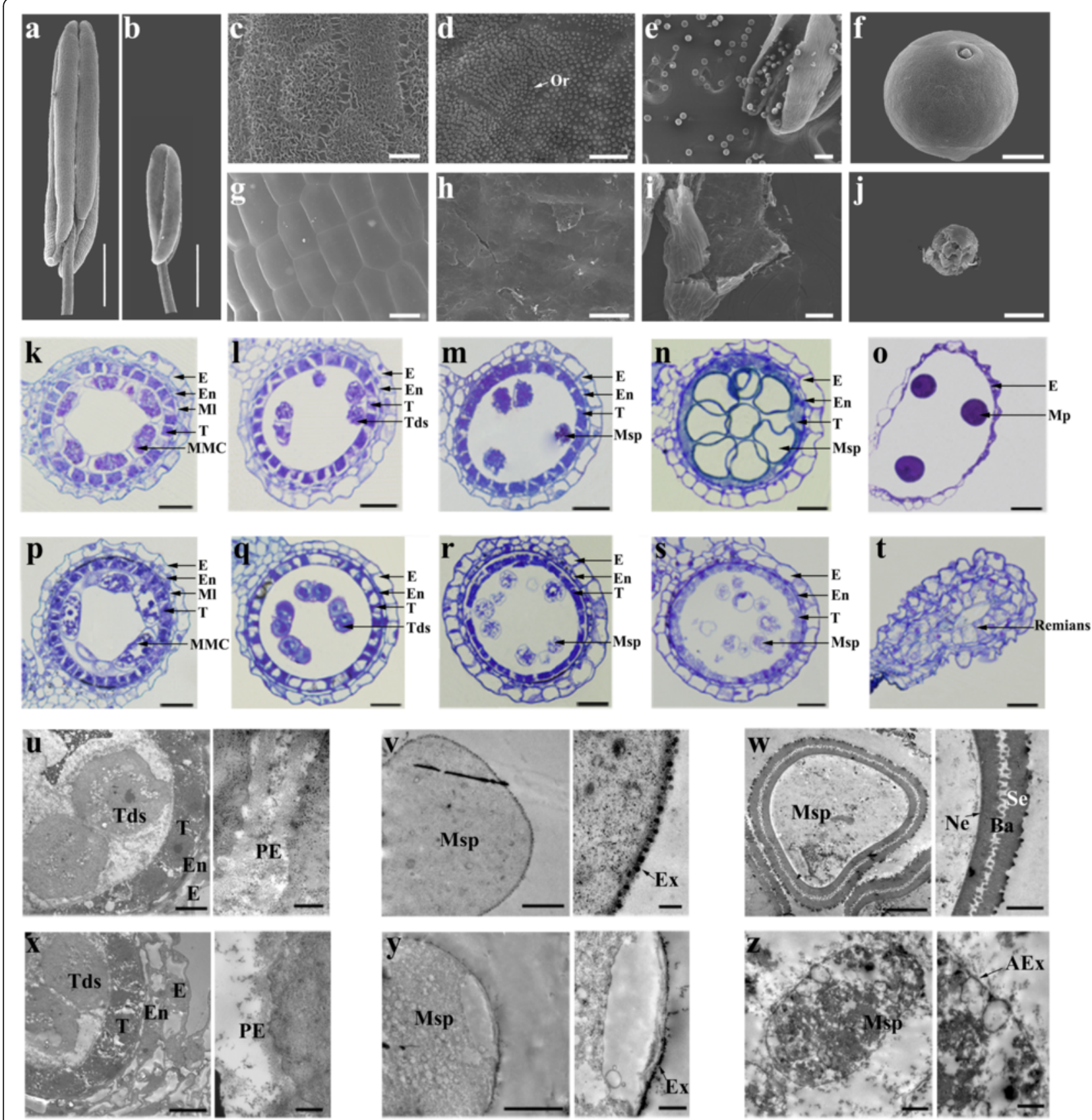

Fig. 3 The defective anther cuticle and pollen sexine formation in osacos12. $\mathbf{a}$ and $\mathbf{b}$ SEM images for the WT and osacos 12 anthers. c-j SEM observation for the epidermal surface of the WT (c) and osacos12 (g) anthers, the inner surface of WT (d) and osacos 12 (h) anthers, and the pollen grains in WT (e and $\mathbf{f}$ ) and osacos12 (i and $\mathbf{j}$ ) anthers. Or, orbicule; Bars $=500 \mu \mathrm{m}$ in $\mathbf{a}$ and $\mathbf{b}, 100 \mu \mathrm{m}$ in $\mathbf{e}, \mathbf{i}, 10 \mu \mathrm{m}$ in $\mathbf{c}, \mathbf{g}, \mathbf{f}, \mathbf{j}$ and $5 \mu \mathrm{m}$ in $\mathbf{h}, \mathbf{d}$. k-t Semi-thin cross-sectional analysis of anther development of WT (k-o) and the osacos 12 mutant (p-t) during the anther development stages. E, epidermis; En, endothecium; ML, middle layer; T, tapetum; MMC, microspore mother cell; Tds, tetrads; Msp, microspore. Bars = 20 um. u-z TEM observation for WT (u-w) and osacos12 (x-z) pollen development from stages 8-10. The boxed image on the right of each panel was enlarged from the left region. AEX, abnormal exine; Ba, bacula; E, epidermis; En, endothecium; Ex, exine; Msp, microspore; Ne, nexine, PE, primexine; Se, sexine; T, tapetum; Tds, tetrads. Bars $=5 \mu \mathrm{m}$ and $500 \mathrm{~nm}$ in $\mathbf{u}, \mathbf{x}, 2 \mu \mathrm{m}$ and $500 \mathrm{~nm}$ in $\mathbf{v}, \mathbf{y}, 5 \mu \mathrm{m}$ and $1 \mu \mathrm{m}$ in $\mathrm{w}, 2 \mu \mathrm{m}$ and $1 \mu \mathrm{m}$ in $\mathbf{z}$

complemented transgenic lines (Fig. 2d). In this transgenic line, OsACOS12-GFP can complement the osa$\cos 12$ phenotype. Thus, the GFP signal in this line represented the OsACOS12-GFP protein level. The GFP signal was detected within the tapetal cells, which formed a circle in the anther (Fig. 5m-o). In the late stages of anther development, the fluorescence signal transferred into the locule (Fig. 5p). However, the GFP 


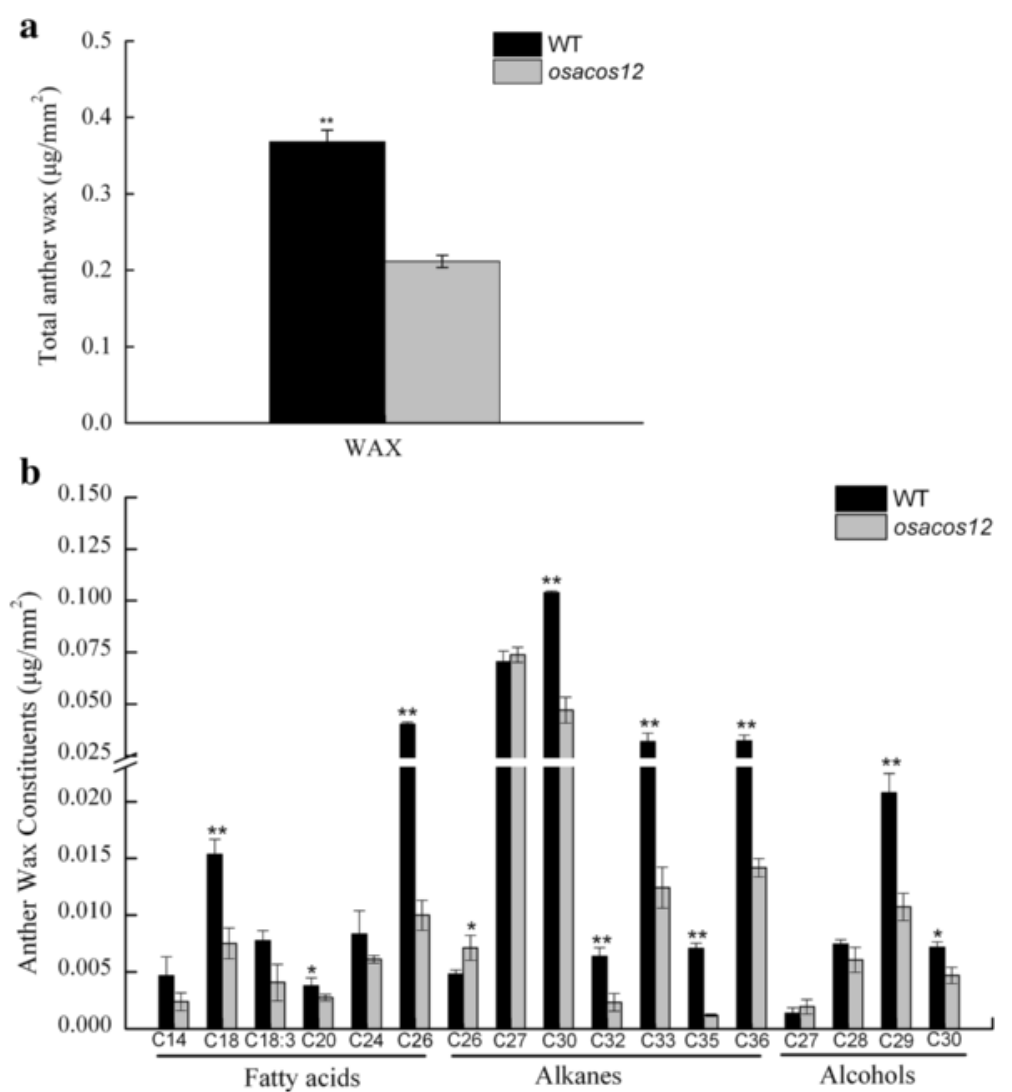

Fig. 4 Anther cuticle wax constitutions in WT and osacos 12. a The total amount of anther wax per unit of anther surface area. $\mathbf{b}$ The amounts of anther wax per unit of anther surface area. Compound names are abbreviated as follows: C14, myristic acid; C18, stearic acid; C18:3, linolenic acid; C20, arachidic acid; C24, lignoceric acid; C26, hexacosanoic acid; C26, hexacosane; C27, heptacosane; C30, triacontane; C32, dotriacontane; C33, tricosane; C35, pentatriacontane; C36, hexatriacontane; C27, 1-heptacosanol; C28, 24-epicampesterol; C29, sitosterol; C30, 1-triacontanol. Values are the mean $\pm \mathrm{SD}(n=3) .{ }^{*}, P<0.05 ;{ }^{*}, P<0.01$ (Student's $t$ test)

signal was not expressed inside the microspores (Fig. 5p). The wild type anther of rice was used as a negative control (Fig. 5k). These results indicate that OsACOS12 is accumulated in tapetal cells and anther locules according to different anther stages.

\section{OsACOS12 could partially fulfil the function of $A \operatorname{COS} 5$ for pollen development in Arabidopsis}

To investigate whether the OsACOS12 and ACOS5 were functionally conserved, genetic complementation of the Arabidopsis acos5 mutant (cs919318, Additional file 3: Figure S3) with the OsACOS12 genomic sequence was performed. We generated two constructs, proACOS5:OsACOS12 and proOsACOS12:OsACOS12, with OsACOS12 driven by ACOS5 and OSACOS12 promoters, respectively. After the constructs were introduced into acos $5 /+$ heterozygous plants (Fig. 6a-b), we obtained 12 and 14 transgenic lines with a homozygous acos 5 background for these constructs (Additional file 4: Figure S4). All 12 transgenic lines for proACOS5:OsACOS12 exhibited partial fertility compared with the complete sterility of $a \cos 5$ (Fig. 6e). RT-PCR demonstrated that OsACOS12 was highly expressed in the transgenic lines (Fig. 6o). Alexander staining showed that these transgenic plants contained mature grains that were similar to the grains of wild type plants (Fig. 6i). However, SEM analysis showed these pollen grains still had slight morphology defects (Fig. 6m). This result showed that the expression of OsACOS12 partially rescued the fertility of the acos 5 mutant, which suggested OsACOS12 can fulfil the function of ACOS5 in Arabidopsis. All the transgenic lines for proOsACOS12:OsACOS12 have complete male sterility (Fig. 6f). RT-PCR demonstrated that the expression of OsACOS12 was low in these transgenic lines (Fig. 60). Alexander staining showed that all pollen grains in the locule were aborted during the late stages of anther development (Fig. 6j). However, SEM showed that many pollen remnants could be formed in the anthers of proOsACOS12:OsACOS12 transgenic plants although these pollen grains were still defective (Fig. 6n). These results suggested that the OsACOS12 promoter was not strong enough to drive the expression of the fatty acyl-CoA synthetase gene in Arabidopsis. 

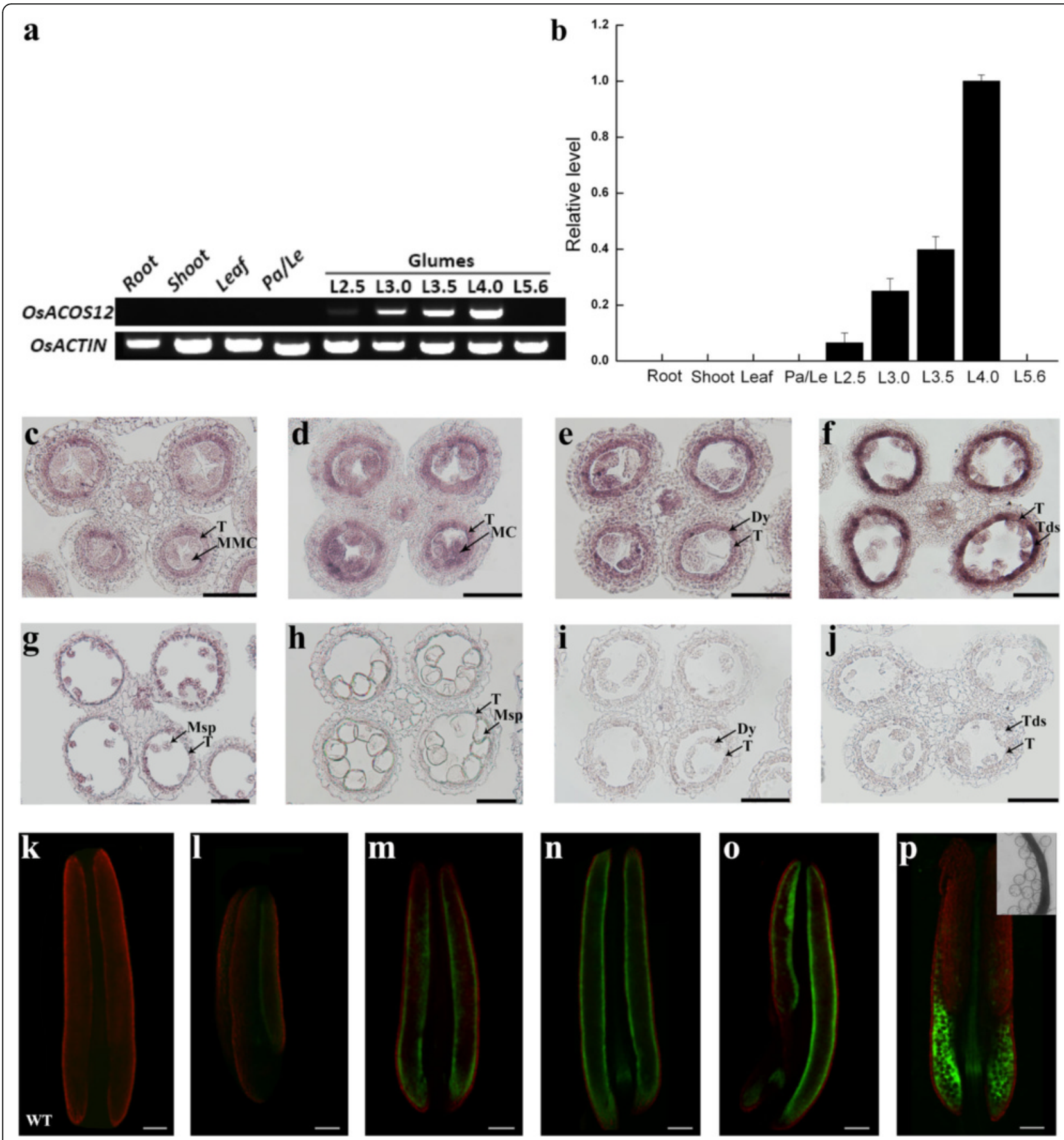

Fig. 5 OsACOS12 is specifically expressed in the anther. a RT-PCR analysis of RNA isolated from various tissues using OSACOS12 and OSACTIN primer sets. Le, lemma; Pa, palea; L2.5, Glumes length 2.5 mm; L3.0, Glumes length 3.0 mm; L3.5, Glumes length 3.5 mm; L4.0, Glumes length $4.0 \mathrm{~mm}$; L5.6, Glumes length $5.6 \mathrm{~mm}$. b Quantitative real-time PCR analysis of OSACOS12. The OSACTIN gene served as the reference. Data are shown as the mean \pm SD $(n=3)$. $\mathbf{c}-\mathbf{j}$ In situ hybridization of OSACOS12 in WT anthers. The anthers at the MMC stage (c), early meiosis stage (d), tetrad stage (e and $\mathbf{f}$ ), microspore release stage $(\mathbf{g})$, and microspore vacuolate stage (h) hybridized with an OsACOS12 antisense probe. The anthers at the tetrad stage (i-j) hybridized with an OsACOS12 sense probe. Msp, microspore; T, tapetum; Tds, tetrads. MMC, microspore mother cell; MC, meiotic cell; Dy, dyad cell. Bars $=50 \mu \mathrm{m}$. k-p Fluorescence confocal images of the OsACOS12-GFP fusion proteins at different stages. The green channel shows the GFP expression $(530 \mathrm{~nm})$, and the red channel shows the chlorophyll autofluorescence $(>560 \mathrm{~nm})$. The bright-field images of (p) show that these fusion proteins are not localized to the microspores. Bars $=10 \mu \mathrm{m} ; 100 \mu \mathrm{m}$ 


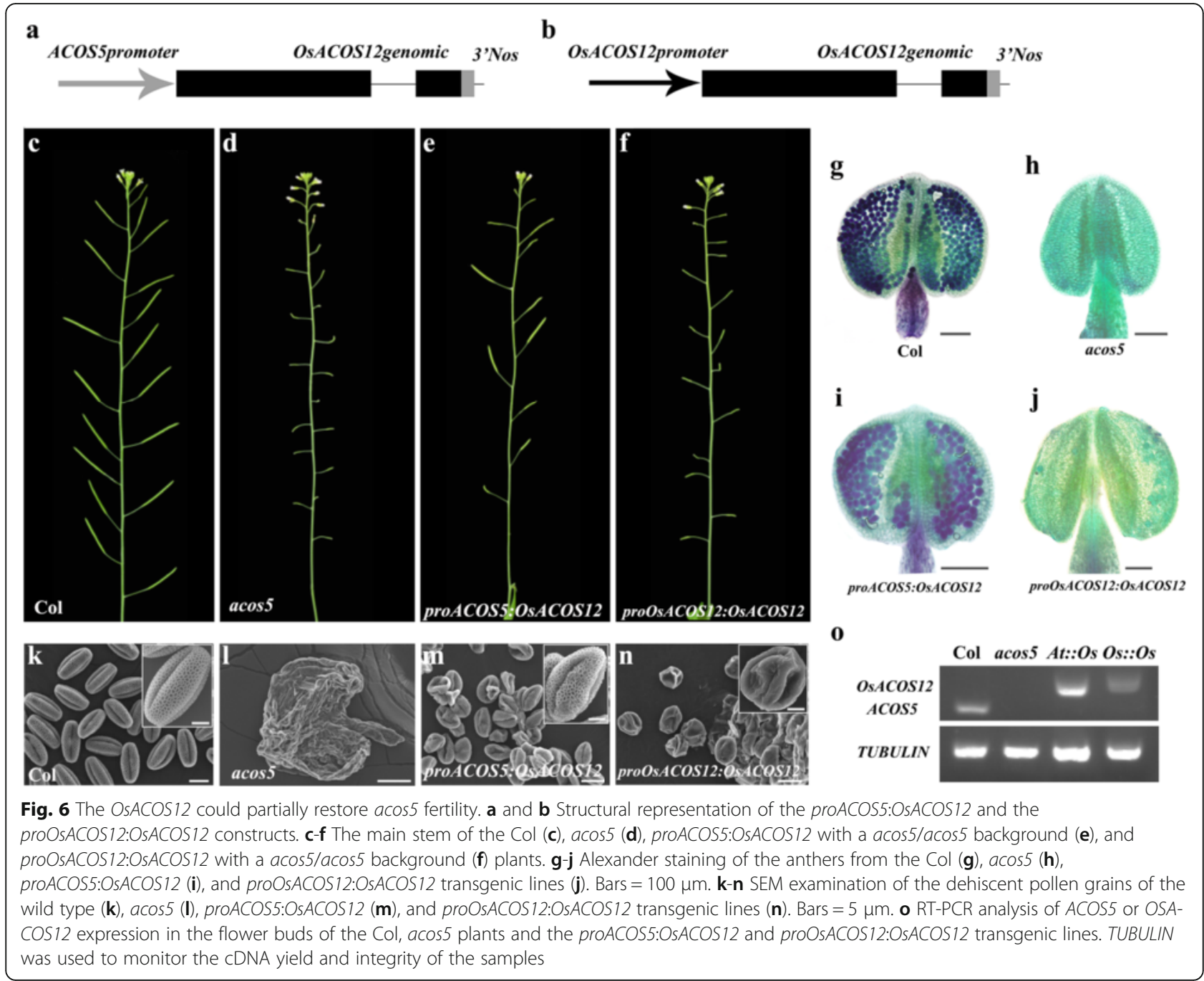

\section{Discussion}

OsACOS12 is an orthologue of Arabidopsis ACOS5 for pollen exine formation and the anther wall in rice

The biosynthesis pathway of sporopollenin precursors has been identified in rice through several genetic studies. The fatty acyl reductase (DPW) converted palmitoylacyl carrier protein (ACP) to palmitoyl alcohol. The WDA1 gene participated in the biosynthesis of very long chain fatty acids. CYP704B2 and CYP703A3 could catalyse the production of w-hydroxylated fatty acids with lauric, palmitic and oleic acid [22-25, 32, 33]. In addition to these catalytic reactions, the fatty acyl-CoAs were indispensable for sporopollenin monomer synthesis. In Arabidopsis, the ACOS5 gene esterifies mediumto long-chain fatty acids to the corresponding fatty acylCoAs for the biosynthesis of sporopollenin [26]. In this study, we identified that OsACOS12 is the orthologue of ACOS5 in rice (Fig. 1). Knockout of OsACOS12 led to the defective exine layer of the microspore and male sterility (Figs. 2 and 3). These results were consistent with the phenotype of acos5 in Arabidopsis [26]. These results suggest that OsACOS12 is involved in the fatty acyl-CoAs synthesis required for sporopollenin precursors. Rice anthers have obvious orbicules and reticulate anther cuticle [24]. The cuticle is essential for rice anther development because it resists abiotic and biotic pressure [34]. Orbicules have been proposed to play a role in the translocation of sporopollenin constituents. The orbicules were barely detected in the inner surface (Fig. 3), and the wax components of the osacos 12 anther were aberrant (Fig. 4). This outcome suggested that OsACOS12 was not only essential for pollen wall formation but also involved in anther cuticle lipid metabolism. The mutants of sporopollenin-related genes including $D P W, C Y P 703 A 3, C Y P 704 B 2, A B C G 15$ in rice also exhibited the defective exine and anther epicuticle formation [22, 24, 25, 32]. This result suggests that the anther cuticle and sporopollenin synthesis share a common lipid metabolism pathway in rice. However, the reticulate anther cuticle was not affected in Arabidopsis 
acos5 mutants (Additional file 5: Figure S5). This result suggests there was a divergence in the lipid pathway between Arabidopsis and rice.

\section{OsACOS12 was expressed in the tapetum and secreted into the locule during pollen development}

The tapetum cell likely synthesizes the sporopollenin precursors and subsequently transports them into the locule to be assembled on the pollen surface and form the sexine layer $[5,8,10,11]$. The ACOS5 is an essential enzyme for sporopollenin synthesis in Arabidopsis. It transcribes components of the tapetum, meiocytes and microspores [17, 26, 35]. OsACOS12 is an orthologue of ACOS5. It exhibits a similar expression pattern to ACOS5 at the transcript level. In the complementary line, the OsACOS12-GFP signal was initially detected in the tapetal cells. At later stages, the signal occupied the free space in the locule but not in the microspores (Fig. 5). During anther development, all tapetal cellular degradation products, including proteins, entered the anther locule after it underwent the PCD process [36]. Given the secretary function of the tapetum, OsACOS12 is likely to be translated in the tapetum and secreted into the locule. Therefore, the OsACOS12 expression pattern at the protein level was different from the transcription level. This trend was also observed for DYT1, an essential regulator for early tapetum development. Its transcript was detected in meiocytes, the tapetum and microspores. However, DYT1 protein was only detected in tapetal cells [37]. The expression pattern of OsACOS12 was consistent with the genes involved in the biosynthesis and transportation processes of sporopollenin precursors. CYP703A2 catalyses the conversion of medium-chain saturated fatty acids to the corresponding monohydroxylated fatty acids in Arabidopsis [13]. It was expressed in the tapetum cells and secreted into the locule at the late stage of anther development [20]. Several lipid transfer proteins (LTPs: LTPC6, LTPC14, OsC6) have been noted for their secretory accumulation pattern $[38,39]$. The LTPs bound or unbound to exine precursors are secreted from the tapetal cells to become exine layer constituents. The protein localization of OsACOS12 and CYP703A2 was different from MS2, which is another enzyme for sporopollenin synthesis in Arabidopsis. It was only localized in tapetal cells [19]. OsACOS12 might be secreted by the tapetum along with sporopollenin transportation.

\section{The sporopollenin biosynthesis pathway was conserved between rice and Arabidopsis}

In Arabidopsis, approximately 9 genes were reported to be involved in sporopollenin biosynthesis and transportation [3]. The orthologues for several genes, including CYP704B2, CYP703A3, OsPKS1, OsTKPR1, DPW and
$A B C G 15$, have been identified in rice [22-25, 32]. In this study, OsACOS12 was identified as an orthologue of ACOS5. OsACOS12 driven by the ACOS5 promoter was able to partially restore the fertility of the male sterile acos5 mutant (Fig. 6e), which suggested that the functions of acyl-CoA synthetases were mainly conserved between monocot and dicot species. Previous studies showed that the rice $D P W$ gene can completely rescue the sexine defects in the $m s 2$ mutant [32]. PpASCL in Physcomitrella patens could produce hydroxyalkyl apyrones, which was consistent with the results from the Arabidopsis orthologue PKSA [40]. It is likely that the functions of the sporopollenin biosynthesis genes were very conservative in land plants. In the transgenic line of proACOS5:OsACOS12, the expression of OsACOS12 was comparable to that of ACOS5 in wild type (Fig. 6o). However, the sexine layer of pollen grains in this transgenic line was still defective (Fig. 6e, m). This result suggests that the enzyme activity of OsACOS12 might be lower than that of ACOS5 in Arabidopsis or that there may be some functional divergence for fatty acid metabolism. The pollen wall patterning for a specific plant species was a conserved and elaborate process [41]. The slightly different substrates and products derived from OsACOS12 probably led to the defective pollen surface in the transgenic line. A recent study also showed that several lipid metabolic enzymes for sporopollenin formation were conserved in tobacco and rice, while some products were different [23]. For the $m s 2$ mutant, PpMS2 driven by the MS2 promoter could not rescue its fertility. However, $D P W$ driven by the MS2 promoter could rescue its fertility with normal pollen wall formation [32, 42]. These results suggested that DPW and MS2 have a very similar function, while PpMS2 and MS2 have evolutionary divergence. In the proOsACOS12:OsACOS12 line, OsACOS12 is driven by its own promoter, and the transgenic plants exhibited a male sterile phenotype. The expression of OsACOS12 in the acos 5 mutant was detected. However, its expression level was lower than ACOS5 in wild type (Fig. 6o). This result suggests that the upstream regulators for sporopollenin synthesis in Arabidopsis could recognize the OsACOS12 promoter. However, the activation efficiency was lower compared to that of the ACOS5 promoter.

\section{Conclusion}

In this study, we functionally identified the OsACOS12 gene in rice, which is an orthologue of Arabidopsis ACOS5. Our results provided genetic evidence to suggest that OsACOS12 was involved in the lipidic metabolism for sporopollenin and cuticle synthesis in rice. The accumulation of OsACOS12 in tapetal cells and the anther locule suggested the processes of sporopollenin biosynthesis and transportation occurred synchronously. Genetic complementation 
assays indicated that $A C O S 5$ and $O s A C O S 12$ were functionally conserved in general for pollen wall formation in rice and Arabidopsis. These findings provide new insights to illustrate fatty acyl-CoA synthetase function in the sporopollenin synthesis pathway of rice and provide a potential male sterile line for the utilization of heterosis in crops.

\section{Methods}

\section{Plant materials and growth conditions}

The osacos12 mutant was obtained from Tilling technology [31]. Rice accessions, including osacos 12 and wild type Zhonghua11 (O. sativa ssp. Japonica), were grown in a paddy field at Shanghai Normal University (Shanghai, China). The Arabidopsis ecotype Columbia- 0 and $a \cos 5$ plants were grown in a growth chamber at DATs of $22{ }^{\circ} \mathrm{C}$ under a $16 \mathrm{~h}$ light and $8 \mathrm{~h}$ dark photoperiod, unless specifically indicated.

\section{Characterization of the mutant phenotype}

The male sterility mutant was crossed with Zhonghua11 to produce the $F_{1}$ generation. The homozygous male sterility mutant was obtained in the $\mathrm{F}_{2}$ generation accompanied by the co-segregation assay. The Arabidopsis and rice florets were photographed with a digital camera (Nikon D7000) and an dissecting microscope (Olympus, SZX10). The wild type and osacos12 mutant anthers were stained with alexander's solution and observed with a Leica microscope (Leica, USA). For a semi-thin section, SEM and TEM observation, spikelets and anthers of wild-type and osacos12 mutants at different stages were dissected to avoid experimental deviation. The embedding and observation procedures were performed as described by Lou et al [2].

\section{Complementation of the $0 s a \cos 12$ and $a \cos 5$ mutants}

For functional complementation, a genomic DNA fragment including the OsACOS12 coding region and 1428 bp promoter sequence were amplified from Zhonghua11 genomic DNA using gene-specific primers (Additional file 6: Table S1). The DNA fragment was subcloned into the pCAMBIA1300 binary vector using the pEASY-Uni seamless cloning and assembly kit with the BamHI restriction enzyme. The construct was introduced into Agrobacterium tumefaciens EHA105 and transformed into the calli induced from osacos 12 heterozygous seeds (Biorun, China). The $\mathrm{T}_{1}$ transgenic lines were genotyped to confirm the homozygous male sterility mutant by PCR and then verified by DNA sequence. OsACOS12-GFP florescence was detected using an LSM 5 PASCAL confocal laser scanning microscope (ZEISS, German).

For the transgenic rescue assay, the coding sequences of the $1047 \mathrm{bp}$ promoter region of ACOS5, $1428 \mathrm{bp}$ promoter region of OsACOS12 and the genomic region of
OsACOS12 were generated by PCR amplification using primer star DNA polymerase (TaKaRa, Japan) and genespecific primers (Additional file 6: Table S1). These sequences were subsequently cloned into the pCAMBIA1300 binary vector (CAMBIA, Australia). Constructs were transformed into fertile heterozygous Arabidopsis acos 5 plants. The transgenic lines were screened using $20 \mathrm{mg} \cdot \mathrm{L}^{-1}$ hygromycin. Genotypic and phenotypic analysis of the segregation populations was then performed in the $\mathrm{T}_{1}$ generation.

\section{Phylogenetic analysis}

The OsACOS12 protein sequence was used to search for orthologues from the plant species using the basic local alignment search tool (BLAST) at the National Center for Biotechnology Information (http://www.ncbi.nlm.nih.gov/ ). Multiple sequence alignments of the full-length protein sequences were performed using ClustalW and displayed using BOXSHADE (http://www.ch.embnet.org/software/ ClustalW.html). The phylogenetic tree was generated by the MEGA6.0 program using the Neighbour-Joining method with default parameters with 1000 bootstrap replicates.

\section{RT-PCR and quantitative real-time PCR assay}

Total RNA from roots, shoots, leaves, paleas and anthers at different developmental stages were extracted using a TRIzol kit (Life Technologies, USA). Subsequently, a $2 \mu \mathrm{g}$ aliquot of total RNA was used as template for reverse transcription by AMV reverse transcriptase with a poly (dT12-18) primer (TOYOBO, Japan). Quantitative PCR analyses were performed with three repeats for each sample using SYBR Green Real-time PCR Master Mix (TOYOBO, Japan) and utilizing an ABI 7300 system (Life Technologies, USA). The quantitative PCR procedure and conditions were described previously by Lou et al [2]. The cDNA levels of the target genes were normalized to the internal standard gene OsACTIN. Three replicates of each sample were used for gene expression analysis. The relevant primer sequences are listed in Additional file 6: Table S1.

\section{In situ hybridization analyses}

Fresh Zhonghua11 young panicles from different developmental stages were fixed in FAA immediately, embedded in paraffin, and sectioned at a thickness of $7 \mu \mathrm{m}$. A $443 \mathrm{bp}$ fragment of OsACOS12 cDNA was amplified from the wild type DNA with its specific primers (Additional file 6: Table S1). The PCR product was cloned into a pBluescript-SK vector (Stratagene) and then digested with BamHI or EcoRI to obtain the templates, respectively. These templates were transcribed in vitro by the T7 or T3 RNA polymerases to produce antisense or sense probes 
(Roche, USA). Tissue embedding, hybridization and signal detection were performed as described by Cai et al [21].

\section{Anther wax quantification}

The anthers at the heading stage from wild type and osa$\cos 12$ mutants were collected for GC-MS analysis with approximately 1800 anthers per sample. Analyses of anther wax constituents and the surface area were performed according to previously described methods [24] with minor modifications. Anthers were extracted with $1 \mathrm{~mL}$ of chloroform for $1 \mathrm{~min}$. The tetracosane $(10 \mu \mathrm{g})$ (Sigma-Aldrich, USA) was added to extract chloroform as an internal standard. The resulting chloroform extract was transferred to a new glass vial and evaporated under a gentle stream of nitrogen. The remaining samples $(100 \mu \mathrm{L})$ were converted with $20 \mu \mathrm{L}$ of bis-(N,N-trimethylsilyl)-tri-fluoroacetamide (Sigma-Aldrich, USA) in $20 \mu \mathrm{L}$ of pyridine for $40 \mathrm{~min}$ at $70^{\circ} \mathrm{C}$ before GC-MS analysis (Agilent GC 6890, DB-1: column $30 \mathrm{~m} \times 0.32 \mathrm{~mm} \times$ $0.1 \mu \mathrm{m}$; on-column injection at $100^{\circ} \mathrm{C}$, oven temperature at $100{ }^{\circ} \mathrm{C}$ for $2 \mathrm{~min}$, increasing at $10{ }^{\circ} \mathrm{C} \cdot \mathrm{min}^{-1}$ to $200{ }^{\circ} \mathrm{C}$, 2 min at $250{ }^{\circ} \mathrm{C}$, increasing at $10{ }^{\circ} \mathrm{C} \cdot \mathrm{min}^{-1}$ to $310{ }^{\circ} \mathrm{C}$, $5 \mathrm{~min}$ at $310^{\circ} \mathrm{C}$ and helium carrier gas at $2 \mathrm{~mL} \cdot \mathrm{min}^{-1}$ ). Quantification of wax compounds was accomplished with the internal standard by integrating the peak areas.

\section{Additional files}

Additional file 1: Figure S1. Complementation of osacos 12 by OsACOS12 genomic. a, e SEM observation for the WT (a) and C-osacos12 (e) anthers. $b$, f The enlarged view of the epidermal surface of WT (b) and Cosacos $12(\mathrm{f})$ anthers. c, g SEM observation for the WT (c) and C-osacos 12 (g) pollen grains. $d, h$ The enlarged view of the epidermal surface of WT (d) and C-osacos 12 (h) pollen grains. Bars $=200 \mu \mathrm{m}$ in a, e, $10 \mu \mathrm{m}$ in b, c, f, g, and $1 \mu \mathrm{m}$ in $\mathrm{d}$, h. (TIF $3787 \mathrm{~kb}$ )

Additional file 2: Figure S2. TEM analysis of the tapetum in the wild type and osacos12. a,c. TEM images for the WT (a) and osacos12 (c) tapetum at stage 8. b, d. TEM images for the WT (b) and osacos12 (d) tapetum at stage 9. T, tapetum. Bars $=2 \mu \mathrm{m}$. (TIF $5079 \mathrm{~kb}$ )

Additional file 3: Figure S3. Gene structure of ACOS 5 and the T-DNA insertion. Exons are shown as black boxes. Introns, promoter, and untranslated regions are shown as lines. T-DNA was inserted in the first exon. LP, Left border primer; RP, right border primer. (TIF $94 \mathrm{~kb}$ )

Additional file 4: Figure S4. RT-PCR analysis of $a \cos 5 / a \cos 5$ background plants using the primer pair LP/RP, LB/RP and LB/LP. (TIF $406 \mathrm{~kb}$ )

Additional file 5: Figure S5. SEM observation of Col and acos 5 mutant anthers. a, d SEM observation for the Col (a) and $\operatorname{acos} 5$ (d) anthers. b and c. An enlarged view of the epidermal surface of the Col anther. e and $f$. An enlarged view of the epidermal surface of the $\operatorname{acos} 5$ anther. Bars $=$ $100 \mu \mathrm{m}$ in a, d, $10 \mu \mathrm{m}$ in b, e and $1 \mu \mathrm{m}$ in c, f. (TIF $2928 \mathrm{~kb}$ )

Additional file 6: Table S1. List of primers used in this study. (DOC $43 \mathrm{~kb}$ )

\section{Abbreviations}

GC-MS: Gas chromatography-mass spectrometry; GFP: Green fluorescent protein; NCBI: National Center for Biotechnology Information; RTPCR: Reverse transcription polymerase chain reaction; SEM: Scanning electron microscopy; TEM: Transmission electron microscopy

\section{Acknowledgements}

We thank Mr. Wenli Hu (Institute of Plant Physiology and Ecology, Shanghai Institutes for Biological Science, Chinese Academy of Sciences) for the GC-MS analysis.

\section{Funding}

This work was supported by grants from the Ministry of Science and Technology of China (2013CB945100) (to Z.N.Y.), the National Key Research and Development Program of China (2016YFD0100902) (to Z.N.Y.) and the National Science Foundation of China (31300262) (to C.Z).

\section{Availability of data and materials}

The data supporting the results of this article are included within the article and its additional files. The gene and promoter sequences of ACOS5 and OsACOS12 were deposited in the TAIR (https://www.arabidopsis.org/) and RGAP (http://rice.plantbiology.msu.edu/) under accession numbers AT1G62940 and LOC_Os04g24530, respectively.

\section{Authors' contributions}

YLL performed most of the experiments, data processing and drafted the manuscript; DDL performed in situ hybridization and cytological analysis; ZLG provided the acos 5 mutant and assisted in complementation; QSS cooperated with YLL in vector construction; SXX provided technical assistance to YLL; CZ revised the manuscript; ZNY and JZ designed and supervised the experiments, and they revised the article. All authors have read and approved this manuscript.

\section{Competing interests}

The authors declare that they have no competing interests.

\section{Consent for publication}

Not applicable.

Ethics approval and consent to participate

Not applicable.

Received: 21 August 2016 Accepted: 3 November 2016

Published online: 21 November 2016

\section{References}

1. Gomez JF, Talle B, Wilson ZA. Anther and pollen development: A conserved developmental pathway. J Integr Plant Biol. 2015;57(11):876-91.

2. Lou Y, Xu XF, Zhu J, Gu JN, Blackmore S, Yang ZN. The tapetal AHL family protein TEK determines nexine formation in the pollen wall. Nat Commun 2014:5:3855

3. Quilichini TD, Grienenberger E, Douglas CJ. The biosynthesis, composition and assembly of the outer pollen wall: A tough case to crack. Phytochemistry. 2015:113:170-82.

4. Jia QS, Zhu J, Xu XF, Lou Y, Zhang ZL, Zhang ZP, Yang ZN. Arabidopsis AThook protein TEK positively regulates the expression of arabinogalactan proteins for nexine formation. Mol Plant. 2015;8:251-60.

5. Scott RJ. Pollen exine: The sporopollenin enigma and the physics of pattern. In: Scott RJ, Stead AD, editors. Molecular and Cellular Aspects of Plant Reproduction. Cambridge, UK: Cambrige University Press; 1994. p. 49-81.

6. Zinkl GM, Zwiebel Bl, Grier DG, Preuss D. Pollen-stigma adhesion in Arabidopsis: A species-specific interaction mediated by lipophilic molecules in the pollen exine. Development. 1999;126:5431-40.

7. Blackmore S, Wortley AH, Skvarla JJ, Rowley JR. Pollen wall development in flowering plants. New Phytol. 2007:174:483-98.

8. Piffanelli P, Ross JH, Murphy D. Biogenesis and function of the lipidic structures of pollen grains. Sex Plant Reprod. 1998;11 (2):65-80.

9. Mariani C, Beuckeleer MD, Truettner J, Leemans J, Goldberg RB. Induction of male sterility in plants by a chimaeric ribonuclease gene. Nature. 1990; 347(6295):737-41.

10. Heslop-Harrison J. Origin of exine. Nature. 1962;195:1069-71.

11. Dickinson HG, Heslop-Harrison J. Common mode of deposition for the sporopollenin of sexine and nexine. Nature. 1968;220:926-7.

12. Ariizumi T, Toriyama K. Genetic regulation of sporopollenin synthesis and pollen exine development. Annu Rev Plant Biol. 2011;62:437-60.

13. Morant M, Jørgensen $K$, Schaller $H$, Pinot F, Møller BL, Werck-Reichhart D, Bak S. CYP703 is an ancient cytochrome P450 in land plants catalyzing in- 
chain hydroxylation of lauric acid to provide building blocks for sporopollenin synthesis in pollen. Plant Cell. 2007;19:1473-87.

14. Dobritsa AA, Shrestha J, Morant M, Pinot F, Matsuno M, Swanson R, Moller BL, Preuss D. CYP704B1 is a long-chain aatty acid-hydroxylase essential for sporopollenin synthesis in pollen of Arabidopsis. Plant Physiol. 2009;151:574-89.

15. Dobritsa AA, Lei Z, Nishikawa S, Urbanczyk-Wochniak E, Huhman DV, Preuss D, Sumner LW. LAP5 and LAP6 encode anther-specific proteins with similarity to chalcone synthase essential for pollen exine development in Arabidopsis. Plant Physiol. 2010;153:937-95.

16. Kim SS, Grienenberger E, Lallemand B, Colpitts CC, Kim SY, Souza Cde A, Geoffroy P, Heintz D, Krahn D, Kaiser M, Kombrink E, Heitz T, Suh DY, Legrand M, Douglas CJ. LAP6/POLYKETIDE SYNTHASE A and LAP5/ POLYKETIDE SYNTHASE B encode hydroxyalkyl a-pyrone synthases required for pollen development and sporopollenin biosynthesis in Arabidopsis thaliana. Plant Cell. 2010;22:4045-66.

17. Grienenberger E, Kim SS, Lallemand B, Geoffroy P, Heintz D, Souza CD, Heitz T, Douglas CJ, Legrand M. Analysis of TETRAKETIDE alpha-PYRONE REDUCTASE function in Arabidopsis thaliana reveals a previously unknown but conserved, biochemical pathway in sporopollenin monomer biosynthesis. Plant Cell. 2010;22:4067-83.

18. Quilichini TD, Friedmann MC, Samuels AL, Douglas CJ. ATP-Binding cassette transporter $\mathrm{G} 26$ is required for male fertility and pollen exine formation in Arabidopsis. Plant Physiol. 2010;154(2):678-90.

19. Chen WW, Yu XH, Zhang KS, Shi JX, De Oliveira S, Schreiber L, Shanklin J, Zhang DB. Male Sterile2 encodes a platis-localized fatty acyl carrier protein reductase requires for pollen exine development in Arabidopsis. Plant Physiol. 2011;157(2):842-53.

20. Xiong SX, Lu JY, Lou Y, Teng XD, Gu JN, Zhang C, Shi QS, Yang ZN, Zhu J. The transcription factor MS188 and AMS from a complex to activate the expression of CYP703A2 for sporopollenin biosynthesis in Arabidopsis thaliana. Plant J. 2016. doi:10.1111/tpj.13284.

21. Cai CF, Zhu J, Lou Y, Guo ZL, Xiong SX, Wang K, Yang ZN. The functional analysis of OsTDF1 reveals a conserved genetic pathway for tapetal development between rice and Arabidopsis. Sci Bull. 2015;60(12):1073-82.

22. Li H, Piont F, Sauveplane V, Werck-Reichhart D, Diehl P, Schreiber L, Franke R, Zhang P, Chen L, Gao YW, Liang WQ, Zhang DB. Cytochrome P450 family member CYP704B2 catalyzes the w-hydroxylation of fatty acids and is required for anther cutin biosynthesis and pollen exine formation in rice. Plant Cell. 2010;22:173-90.

23. Wang YB, Lin YC, So J, Du YG, Lo C. Conserved metabolic steps for sporopollenin precursor formation in tobacco and rice. Physiol Plantarum. 2013;149(1):13-24.

24. Qin P, Tu B, Wang YP, Deng LH, Quilichini TD, Li T, Wang H, Ma BT, Li SG. ABCG15 encodes an ABC transporter protein, and is essential for PostMeiotic anther and pollen exine development in rice. Plant Cell Physiol. 2013;54(1):138-54.

25. Yang XJ, Wu D, Shi JX, He Y, Pinot F, Grausem B, Yin CS, Zhu L, Chen MJ, Luo ZJ, Liang WQ, Zhang DB. Rice CYP703A3, a cytochrome P450 hydroxylase, is essential for development of anther cuticle and pollen exine. J Integr Plant Biol. 2014;56(10):979-94.

26. de Azevedo SC, Kim SS, Koch S, Kienow L, Schneider K, McKim SM, Haughn GW, Kombrink E, Douglas CJ. A novel satty acyl-coA synthetase is required for pollen development and sporopollenin biosynthesis in Arabidopsis. Plant Cell. 2009;21:507-25.

27. Lallemand B, Erhardt M, Heitz T, Legrand M. Sporopollenin biosynthetic enzymes interact and constitute a metabolon localized to the endoplasmic reticulum of tapetum cells. Plant Physiol. 2013;162(2):616-25.

28. de Azevedo SC, Barbazuk B, Ralph SG, Bohlmann J, Hamberger B, Douglas CJ. Genome-wide analysis of a land plant-specific acyl:coenzymeA synthetase (ACS) gene family in Arabidopsis, poplar, rice and Physcomitrella. New Phytol. 2008;179:987-1003.

29. Shockey JM, Fulda MS, Browse J. Arabidopsis contains a large superfamily of acyl-activating enzymes. Phylogenetic and biochemical analysis reveals a new class of acyl-coenzyme a synthetases. Plant Physiol. 2003;132(2):1065-76.

30. Guo XJ, Jiang ML, Wan X, Hu CJ, Gong YM. Identification and biochemical characterization of five long-chain acyl-coenzyme a synthetases from the diatom Phaeodactylum tricornutum. Plant Physiol Bioch. 2014;74:33-41.

31. Wang T, Uauy C, Till B, Liu CM. TILLING and associated technologies. J Integr Plant Biol. 2010;52(11):1027-30.

32. Shi J, Tan HX, Yu XH, Liu YY, Liang WQ, Ranathunge K, Franke RB, Schreiber L, Wang YJ, Kai GY, Shanklin J, Ma H, Zhang DB. Defective pollen wall is required for anther and microspore development in rice and encodes a fatty acyl carrier protein reductase. Plant Cell. 2011;23:2225-46.

33. Jung KH, Han MJ, Lee DY, Lee YS, Schreiber L, Franke R, Faust A, Yephremov A, Saedler H, Kim YW, Hwang I, An G. Wax-deficient anther1 Is Involved in Cuticle and Wax Production in Rice Anther Walls and Is Required for Pollen Development. Plant Cell. 2006;18:3015-32.

34. Bai J, Zhu X, Wang Q, Zhang J, Chen H, Dong G, Zhu L, Zheng H, Xie Q, Nian J, Chen F, Fu Y, Qian Q, Zuo J. Rice TUTOU1 Encodes a Suppressor of CAMP Receptor-Like Protein That Is Important for Actin Organization and Panicle Development. Plant Physiol. 2015;169:1179-91.

35. Quilichini TD, Douglas CJ, Samuels AL. New views of tapetum ultrastructure and pollen exine development in Arabidopsis thaliana. Ann Bot. 2014;114:1189-201.

36. Wu HM, Cheung AY. Programmed cell death in plant reproduction. Plant Mol Bio. 2000:44:267-81.

37. Gu JN, Zhu J, Yu Y, Teng XD, Lou Y, Xu XF, Liu JL, Yang ZN. DYT1 directly regulates the expression of TDF1 for tapetum development and pollen wall formation in Arabidopsis. Plant J. 2014;80(6):1005-13.

38. Zhang DH, Liang WQ, Yin CS, Zong J, Gu FW, Zhang DB. OsC6, encoding a lipid transfer protein, is required for postmeiotic anther development in rice. Plant Physiol. 2010;154(1):149-62.

39. Huang MD, Chen TLL, Huang AHC. Abundant type III lipid transfer proteins in Arabidopsis tapetum are secreted to the locule and become a constituent of the pollen exine. Plant Physiol. 2013;163(3):1218-29.

40. Colpitts CC, Kim SS, Posehn SE, Jepson C, Kim SY, Wiedemann G, Reski R, Wee AG, Douglas CJ, Suh DY. PpASCL, a moss ortholog of anther-specific chalcone synthase-like enzymes, is a hydroxyalkylpyrone synthase involved in an evolutionarily conserved sporopollenin biosynthesis pathway. New Phytol. 2011;192(4):855-68.

41. Xu T, Zhang C, Zhou Q, Yang ZN. Pollen wall pattern in Arabidopsis. Sci Bull. 2016;61(11):832-7.

42. Wallace $S$, Chater CC, Kamisugi Y, Cuming AC, Wellman CH, Beerling DJ, Fleming AJ. Conservation of Male Sterility 2 function during spore and pollen wall development supports an evolutionarily early recruitment of a core component in the sporopollenin biosynthetic pathway. New Phytol. 2015;205(1):390-401

\section{Submit your next manuscript to BioMed Central and we will help you at every step:}

- We accept pre-submission inquiries

- Our selector tool helps you to find the most relevant journal

- We provide round the clock customer support

- Convenient online submission

- Thorough peer review

- Inclusion in PubMed and all major indexing services

- Maximum visibility for your research

Submit your manuscript at www.biomedcentral.com/submit
( ) BioMed Central 